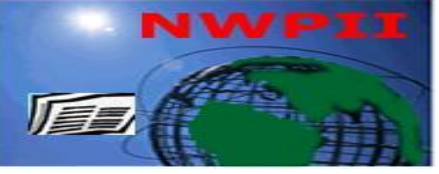

American Journal of Biomedical Sciences

ISSN: 1937-9080

nwpii.com/ajbms

\title{
Evaluation, Characterization and Cell Viability of Ceramic Scaffold and Nano-gold loaded Ceramic Scaffold for Bone Tissue Engineering
}

\author{
Ahmed Farag Aly ${ }^{1}$, Amal S. Eldesouky ${ }^{1}$ and Kamel A.M.Eid ${ }^{2 *}$ \\ ${ }^{1}$ Department of Biomedical Engineering, Helwan University, EGYPT. \\ ${ }^{1}$ Department of Biomedical Engineering, HTI, EGYPT. \\ ${ }^{2}$ Department of Chemistry, Helwan University, Egypt. \\ *Corresponding Author: \\ Kamel A.M. Eid \\ Researcher Assistant Novel Diagnostics \& Therapeutics Research Group \\ School of Sciences \& Engineering \\ The American University In Cairo \\ AUC Avenue, SSE \# 1184, P.O. Box 74 \\ New Cairo, Egypt 11835 \\ Office:+2022615-2565 \\ Cell: +201113008843 \\ Fax: +20227957565 \\ E-mail: kamelam@aucegypt.edu
}

Received:9 March 2012; | Revised: 19 July 2012; | Accepted: 5 September 2012

\begin{abstract}
Orthopaedic implants and metal implantation are major technological contributions in the field of orthopaedic surgery. However, bacterial infection and inflammation are predicament issues that subsequently lead to implant failure and second surgery. Ceramic scaffold loaded with gold nanoparticles (Au NPs) posse's antimicrobial and anti-inflammatory properties, which would be more ideal for successful bone implantation and tissue regeneration. Thereby, Hydroxyapatite nanoparticles (nHA), $\beta$-Tricalcium Phosphate nanoparticles (n $\beta$-TCP), and Au NPs were used for the fabrication of ceramic scaffold and Au NPs loaded ceramic scaffold. The effects of the Au NPs on the scaffold's mechanical properties, porosity and cell growth have been evaluated. Scanning Electron Microscope [1] and test metric universal testing machine were employed for characterization of the scaffolds. Gold loaded scaffold demonstrated enhanced porosity, degradability and mechanical properties compared with the ceramic scaffold. The porosity of the ceramic and $\mathrm{Au}$ NPs loaded ceramic scaffold ranged between 30-50\% and 60-75\%, respectively, while compressive strength ranged between $10-30 \mathrm{mPa}$ and $25-45 \mathrm{mPa}$, respectively.. Scaffold synthesis can be used for implantation in organs that need high load bearing such as femurs, tibia and also as a substrate for Au NPs delivery. To our knowledge, Au NPs have not been incorporate previously with calcium phosphate for fabrication scaffold for bone grafting. Also this study the first report on the effects of Au NPS on the mechanical properties, porosity and degradation rates of ceramic scaffolds.
\end{abstract}


Keywords: Gold Nanoparticles, Tissue Engineering, Bone Grafting, Hydroxyapatites and Calcium Phosphate.

\section{Introduction}

Tissue engineering is a fast growing field that provides unique alternative solutions for organ transplants, and thus offers dramatic potential improvement in human health. Autologous and allograft techniques for bone grafting are the most common choice for filling large bone defects [2]. However, these approaches have their drawbacks, such as the need for second surgery at the donor site, limited quantity and shape of available bone, and resorption of the bone graft. A range of porous materials, such as ceramic, polymer and metal, have been developed in the field of bone grafts and scaffolds for tissue engineering [2]. Some of the most favourable bioactive ceramics materials that were used in bone grafting and tissue engineering are hydroxyapatite (nHA) and $\beta$ tricalcium phosphate (n $\beta-T C P)$ [3]. Both materials enhance the mechanical properties, biological properties, and protein absorption of the composite used for scaffold synthesis. Consequently, they have been extensively used in biomedical implants for bone regeneration, such as coating for metallic implants, bone grafts, and composites for middle bone implants [4-5]. In addition to their biocompatibility and biodegradability, the osteoconductive properties of these composites make them ideal materials for bone regeneration; however, their poor mechanical properties, in particular their toughness, is a significant problem. Successful incorporation of nHA and nTCP with polymers has been achieved for the fabrication of scaffolds that have good porosity, degradability and protein absorption, while accomplishing acceptable mechanical properties in term of toughness [6]. These scaffolds were subsequently used as an alternative autologous bone graft for tissue engineering [710]. Generally, tissue engineering scaffolds should have suitable pore area micro-pore size, degradability and good mechanical properties. Designing a scaffold material to mimic the structure and properties of tissue to be replaced offers a tremendous potential for solving these problems [11-12].

Noble metals nanoparticles have received much attention in synthesis methods [13-14], with various morphologies and have been successfully introduced in various applications, such as antimicrobial [15]. Despite their exceptional physical, chemical, and mechanical properties, noble metals nanoparticles generally require surface modification to create favourable biological responses [16]. Au NPs have demonstrated a profound impact on a variety of applications, including drug activity enhancement, DNA detection, medical diagnostics, antimicrobial and surface-enhanced plasmon sensing [17-19].

$\mathrm{Au}$ NPs have been successfully synthesised with hydoxyapatitee for low platelet adhesion and was shown to enhance blood compatibility [20-21], but surface treatment for $\mathrm{Au}$ NPs is needed for cytotoxicty reduction [21].

Accordingly, combining cells capable of osteogenetic activity with an optimum scaffolding material to stimulate bone regeneration and repair remains be done. In particular, osteoblast stem cells have a high proliferation capacity, multiline age potential and high differentiation rates into osteoblast lick bone when seeded within bone scaffold [22]. Herein, we focused on the synthesis of a highly porous, interconnected pore, biodegradable, high load bearing, and safe ceramic scaffold and Au NPs loaded ceramic scaffold that can be used for bone healing and tissue regeneration. The effects of Au NPs and ceramic on the mechanical properties of scaffold, porosity, and cell adhesion and cell viability have been studied.

\section{Materials and Methods}

\subsection{Materials}

Tetra aureate chloride hydride Hydrochloroauric acid trihydrate $\left(\mathrm{HAuC}_{14}{ }_{33} \mathrm{H}_{2} \mathrm{O}\right.$, 99.9\%), tri-sodium citrate $\mathrm{Na}_{3} \mathrm{C}_{6} \mathrm{H}_{5} \mathrm{O}_{7}$, Maconci agar medium, Agarized Czapek Dox, Dulbecco's Modification of Eagles Med (DMEM), glutamine, penicillin, streptomycin and MTT dye, all were 
provided by Sigma Aldrich (Germany). Diammonium hydrogen phosphate powder $\left(\left(\mathrm{NH}_{4}\right)_{2} \mathrm{PO}_{4}\right)$, Calcium nitrate $\left(\mathrm{Ca}\left(\mathrm{NO}_{3}\right)_{2}\right)$ powder, Ammonium Soln $\left(\mathrm{NH}_{4} \mathrm{OH}\right)$, Carboxy methyl Cellulose (CMC), Poly Carbonate Filter paper, Hydrogen chloride $(\mathrm{HCl})$, Nitric acid $\mathrm{HNO}_{3}$ and deionized water were provided by El Gomhoria Co. (Egypt). All chemicals were used without additional purification.

\subsubsection{Synthesis of gold nanoparticles}

Gold nanoparticles have been synthesized using the reported citrate reduction method [23] with modification. Briefly, 3-neck round bottom flask $(100 \mathrm{~mL})$ were cleaned with aqua regia (3 parts $\mathrm{HCl}, 1$ part $\mathrm{HNO}_{3}$ ) and rinsed with deionised water. $\mathrm{HAuCl}_{4}$ solutions $(1 \mathrm{mM}, 100 \mathrm{~mL})$ were heated to boiling and refluxed while being constantly stirred. Then different concentrations of tri-sodium citrate solutions ranged from 38, 40, and 45 and $50 \mathrm{mM}(10 \mathrm{~mL})$ were added in situ. The solution colour had been noticed to change from yellow to black and finally to deep red. After the colour changed, the solution has been refluxed for an additional $15 \mathrm{~min}$. The heat was then turned off and the solution had been stirred until it cooled to room temperature.

\subsubsection{Synthesis of hydroxyapatite nanoparticles}

Hydroxyapatite (nHA) was prepared using the chemical Co-precipitation method [24-25]. Briefly, $0.6 \mathrm{M}$ of $\left(\mathrm{NH}_{4}\right)_{2} \mathrm{PO}_{4}$ were added to DI water to make a $500 \mathrm{ml}$ solution of diammonium hydrogen phosphate. $500 \mathrm{ml}$ of $1 \mathrm{M}$ calcium nitrate solution was prepared by dissolving in DI water. The $\mathrm{pH}$ of the solution was adjusted to 11 by addition of $\mathrm{NH}_{4} \mathrm{OH}$. White precipitates of hydroxyapatite were formed by adding the diammonium hydrogen phosphate solution into the calcium nitrate solution by dropping under constant stirring for a one hour. The solution was reflux for one hour and aged for one day. The precipitates washed with DI water and dried at $80^{\circ} \mathrm{C}$ for another day then calcinated at $800^{\circ} \mathrm{C}$ for one hour by $10^{\circ} \mathrm{C}$ per minute and then cooling to room temperature by $10^{\circ} \mathrm{C}$ per minute.

\subsubsection{Synthesis of $\beta$-Tri-calcium Phosphate}

Tricalcium phosphate (n $\beta-\mathrm{TCP})$ was prepared using the chemical precipitation method [26]. Briefly, a solution containing $0.4 \mathrm{M}$ of $\left(\mathrm{NH}_{4}\right)_{2} \mathrm{PO}_{4}$ was added to a calcium nitrate solution containing $0.6 \mathrm{M}$ of $\mathrm{Ca}\left(\mathrm{NO}_{3}\right)_{2} .4 \mathrm{H}_{2} \mathrm{O}$ to make $1000 \mathrm{ml}$ of solution. The $\mathrm{pH}$ of the solution was adjusted to 10 by addition of $\mathrm{NH}_{4} \mathrm{OH}$. White precipitates were formed in the solution after it aged for one day. The precipitates were washed with DI water and ethanol dried at $80^{\circ} \mathrm{C}$ for another day, then calcinated at $900^{\circ} \mathrm{C}$ for two hours by incrementing $10^{\circ} \mathrm{C}$ per minute and then cooling to room temperature by decrementing $10^{\circ} \mathrm{C}$ per minute.

\subsubsection{Fabrication of Ceramic Scaffold (nHA- n $\beta$-TCP)}

The nHA-n $\beta$-TCP ceramic scaffold was fabricated using heat sintering. Briefly, nHA was added to $\mathrm{n} \beta$-TCP with molar ratio $3: 3$, and with concentrations of $20 \%, 30 \%$ and $50 \%$, consecutively. Next, the powder was added to poly vinyl alcohol solutions (10 wt \%) to create ceramic slurries. A solution of $5 \mathrm{ml}$ of $2.5 \%$ glutaraldehyde was then added drop-wise as a cross-linker. Finally, the slurries were cast into a mould and dried at $100^{\circ} \mathrm{C}$ for 24 hours and then sintered at $350^{\circ} \mathrm{C}$ by $10^{\circ} \mathrm{C}$ per minute for 8 hours. The moulds were allowed to cool at a rate of $10^{\circ} \mathrm{C}$ per minute, until they reached room temperature.

\subsubsection{Synthesis of Ceramic Gold_Scaffold}

The Au NPs loaded ceramic scaffolds were fabricated by heat sintering. Briefly, nHA was added to nTCP with molar ratio $3: 3$ in a concentration of $50 \%$. The powder was added to poly vinyl alcohol solution ( $10 \%$ by wt) to create ceramic slurries and CMC ( $2 \%$ by wt). Next, a Au NPs solution of $50 \mathrm{ml}$ and $0.1 \mathrm{M}$ with concentrations of 5, 10 and $15 \%$ were added to the slurries mixture with slow stirring and heating to complete mixing. As a cross-linker, a solution of 2 $\mathrm{ml}$ of $2.5 \%$ glutaraldehyde was added drop wise. Finally the slurries were cast into a mould and dried at $100^{\circ} \mathrm{C}$ for 24 hours and then sintered at $350^{\circ} \mathrm{C}$ by incrementing $10^{\circ} \mathrm{C}$ per minute for 8 hours. The moulds were allowed to cool at a rate of $10^{\circ} \mathrm{C}$ per minute until it reached to room temperature. 


\subsubsection{Seeding Cell}

Scaffolds of size $14 \mathrm{mmx} 10 \mathrm{~mm}$ were seeded with culture medium human osteoblast cells for a period of 26 days. The scaffolds were submerged in $70 \%$ ethanol for sterility and autoclaved for 2 hours at $120^{\circ} \mathrm{C}$. They were then washed with phosphate buffered saline (PBS 7.4) and soaked in $5 \mathrm{ml}$ of culture medium for $1 \mathrm{~h}$.

Human osteoblast cells were grown in 24 well plates at a density of $5 \times 10^{6}$ cells $/ \mathrm{ml}$ in Medium (DMEM) containing fetal bovine serum $(10 \% \mathrm{~V} / \mathrm{V})$ glutamine $(2 \mathrm{mM})$, penicillin (100 $\mathrm{U} / \mathrm{mL})$ and streptomycin $(1 \mu \mathrm{g} / \mathrm{mL})$, and incubated at $5 \% \mathrm{CO} 2$ at $37^{\circ} \mathrm{C}$. The culture mediums were changed every 48 hours for a period of 26 days.

\subsection{Characterization of Composite Scaffold}

\subsubsection{Scaffold Morphology}

Mmicrostructures and morphology of the scaffolds were examined at 10kV under JXA-840A SEM probe analyzer (Jeol, Japan). Samples were bleached, cut with a micrometer, mounted on cupper stubs and coated with gold using coated sputter (S150A Edward, England).

\subsubsection{In vitro Biological Test}

Cell viability, cell proliferation and cell adhesion were evaluated via SEM. Cell seeded scaffolds were prepared for SEM observation by changing culture medium, washing 3 times in pre warmed PBS 7.4, and observed by SEM on days 14, 21 and 26. After thorough washing with PBS, the specimens were fixed in $2.5 \%$ glutaraldehyde at $4{ }^{0} \mathrm{C}$ for 4 hours and dehydrated in ascending grades of ethanol solutions (50, 70, 90 and 97\%), where each step lasted $30 \mathrm{~min}$. Finally, the samples were left to dry over a 2 hour period, mounted on aluminum stubs coated with carbon tape and examined by Environmental SEM under 10, 15, and $30 \mathrm{kV}$ (SEM XL-1 Philips, Tokyo, Japan).

\subsubsection{Porosity Measurements}

Porosity measurements were determined by liquid displacement [27]. A sample of measured weight, W, was put into a graduated cylinder containing a known volume $\left(\mathrm{V}_{1}\right)$ of ethanol $\left(\right.$ density $\left.=0.789 \mathrm{~g} / \mathrm{cm}^{3}\right)$. The cylinder was then put under vacuum to extract the air out of the scaffold and force ethanol into the space of the scaffold. The vacuum process continued until no air bubbles were observed emerging from the scaffold. The total volume of ethanol and the ethanol-impregnated scaffold was then recorded as $\mathrm{V}_{2}$ by reading the level in the graduated cylinder. The volume difference $\left(\mathrm{V}_{2}-\mathrm{V}_{1}\right)$ represented the volume of the scaffold. The ethanol-impregnated scaffold was removed from the cylinder and the residual ethanol volume was recorded as $\mathrm{V}_{3}$. Thus, the total volume of the scaffold was calculated as $\mathrm{V}=\mathrm{V}_{2}-\mathrm{V}_{3}$.

To calculate the pore volume of the scaffold the initial weight of the scaffold, $\mathrm{W}_{\mathrm{i}}$, and its final weight, $\mathrm{W}_{\mathrm{f}}$, were measured. After utilization of the mean value of triplicate measurements, the porosity was calculated using the following equation:

Porosity $=\left\{\left(\mathrm{W}_{\mathrm{f}}-\mathrm{W}_{\mathrm{i}}\right) / \mathrm{P}_{\text {ethanol }}\right\} / \mathrm{V}$ Eq. (1)

\subsubsection{In vitro Degradation Test of the scaffold}

In vitro degradation of the scaffolds were investigated by soaking in $\mathrm{SBF}(\mathrm{PH} 7.4)$ at $37^{\circ} \mathrm{C}$ for 30 days, where the ratio of solution volume to scaffold mass was $200 \mathrm{ml} / \mathrm{g}$. After soaking, the scaffolds was dried at $120^{\circ} \mathrm{C}$ for 1 day and the rates of weight loss of the scaffolds were measured with different $\mathrm{Au}$ NPs contents as a function of soaking time in SBF [28].

\subsubsection{Mechanical test}

To measure mechanical strength in terms of compressive force, the scaffolds were cut into uniform sized blocks of $20 \mathrm{~mm}$ diameter and $20 \mathrm{~mm}$ height utilizing a micrometer. The scaffold's compressive was tested using a test metric universal testing machine (AG-10AT, Japan) by applying uniaxial compressive force for $80 \%$ deformation. The rate of strain was fixed by adjusting the cross head speed to $1 \mathrm{~mm} / \mathrm{min}$. Data points of force, distance and time were collected and analyzed for stress and strain relationships. Since the cross sectional area of the compressed cellular solids did not expand significantly, for a true stress calculation the cross sectional area of the solid was treated as equal at all points during compression. Thus, the results can be counted as 
true stress, and the stress/strain data was computed and plotted.

\section{Result and Analysis}

\subsection{SEM Analysis}

The size and morphology of the HA, TCP, Au NPs were studied using SEM, and the particle size was obtained using image analysis. To get the distribution of particle size, the diameter of randomly selected particles from the different visions of each sample was measured. As shown in Fig 1A-C, the size is reported as the mean diameter. It is clearly apparent that the HA, TCP and $\mathrm{Au}$ NPs nanoparticles have rod, crystal and spherical like shape, respectively, with a well controlled particle size. Also, the particle size strongly depends preparation conditions, as would be expected. As the distribution of particles size of HA, TCP, Au NPs became narrower, the average particle size became smaller. An average particle size about $50-100 \mathrm{~nm}, 100-130 \mathrm{~nm}$, and $5-10 \mathrm{~nm}$, for HA, TCP and Au NPs, respectively, were observed in the samples as shown in Fig 1 A-C. These results are in agreement with other nanosuspension systems described elsewhere [3, 23, 26].

The fabricated ceramic scaffold displayed a good porous but less interconnected open pore structure, while the Au NPs loaded scaffold displayed a highly porous and interconnected open pore structure. Ceramic scaffolds had pore size distribution ranging between 50-400 micrometers, as shown in Fig.2 A-C. On the other hand, $\mathrm{Au}$ NPs loaded ceramic scaffolds had pore size distribution in range of 100-600 micro-meters, as shown in Fig. 2 D-F.

The observed final porous structures of the scaffold are clearly a function of Au NPs and ceramic concentration. Thus, it may be deduced that increasing Au-HA-TCP concentrations increase both of the porosity and interconnectivity of the scaffolds, while increasing the concentration of HA-TCP without Au NPs reduces porosity and interconnectivity. The Interconnected pore structure is important to bone cell growth, tissue regeneration and interface support. Furthermore, the micro-porous structure is beneficial to capillary growth, nutrient transport and biological properties of the implant [10-11].

\subsection{In vitro Biological Testing \& Imaging}

SEM studies depicted good cell adhesion over the scaffolds materials. After 14 days of growing in culture, osteoblast cells differentiated inside the culture pores to semi confluence on the scaffold. The densely populated cells appeared to be growing in multiple layers, as shown in Fig.3- A and B. Highly connected cell sheet and network like appearance, without pore occlusion and pore bridging, were observed to cover pores with diameter size less than $200 \mu \mathrm{m}$. SEM images obtained on day 21 demonstrated extra-cellular matrix deposition, as shown in Fig.3-C and 3-D, where cells had merged to form a uniform cell matrix like canopy over the scaffold.

Interestingly, cells attached well on the wall of the scaffolds, Moreover, the matrix was seen oriented along the pores making circular regions similar to natural bone, and closely followed the contours. Furthermore, the visible cells on the scaffold were observed to have penetrated and completely covered the pores, apparent from SEM images obtained on day 26 (Fig. 3-E-F).

As the cell migrated into the scaffold, they attached themselves closely to the scaffold walls. Also, the cells not only lined to the wall of tunnels in the porous structure, but also stretched themselves out across the diameter of tunnels and completely covered it. The porous surface improves the mechanical interlocking between the implant and the surrounding natural bone, providing greater mechanical stability at the critical interface [6].

Most importantly, the cell culture results clearly indicated that the Au NPs loaded scaffold is a safe material to use, as it promoted good cell attachment and allowed cell proliferation. Additionally, the chance of cells in the culture to cover up pores and block up the tunnels that were less than $500 \mu \mathrm{m}$ in diameters was high, which is of concern for both in vitro and in vivo situations. It should be also noted that the safe and effective effects of $\mathrm{Au}$ NPs content on the bone cell activity and osteogenic differentiation. Therefore, ceramic Au NPs loaded scaffold possesses the potential to act as a safe supporter cells carrier in orthopedic 
transplants, because of the incorporation of cells transforms it into a safe osteoinductive Implant [29]. Rather than relying on the biodegradable scaffold alone, the enhanced mechanical and biological properties of Au NPs loaded scaffold will definitely be useful when the portion of bone to regenerate is large. In addition to the previous, the $\mathrm{Au}$ NPs content with ceramic scaffold will prevent the invasion of vascular networks, nutrients and gaseous transportation from surrounding tissue via anti-inflammatory effects [30], which avoids a build up of metabolic waste, cell necrosis and an inflammatory response when implanted into a patient [31].
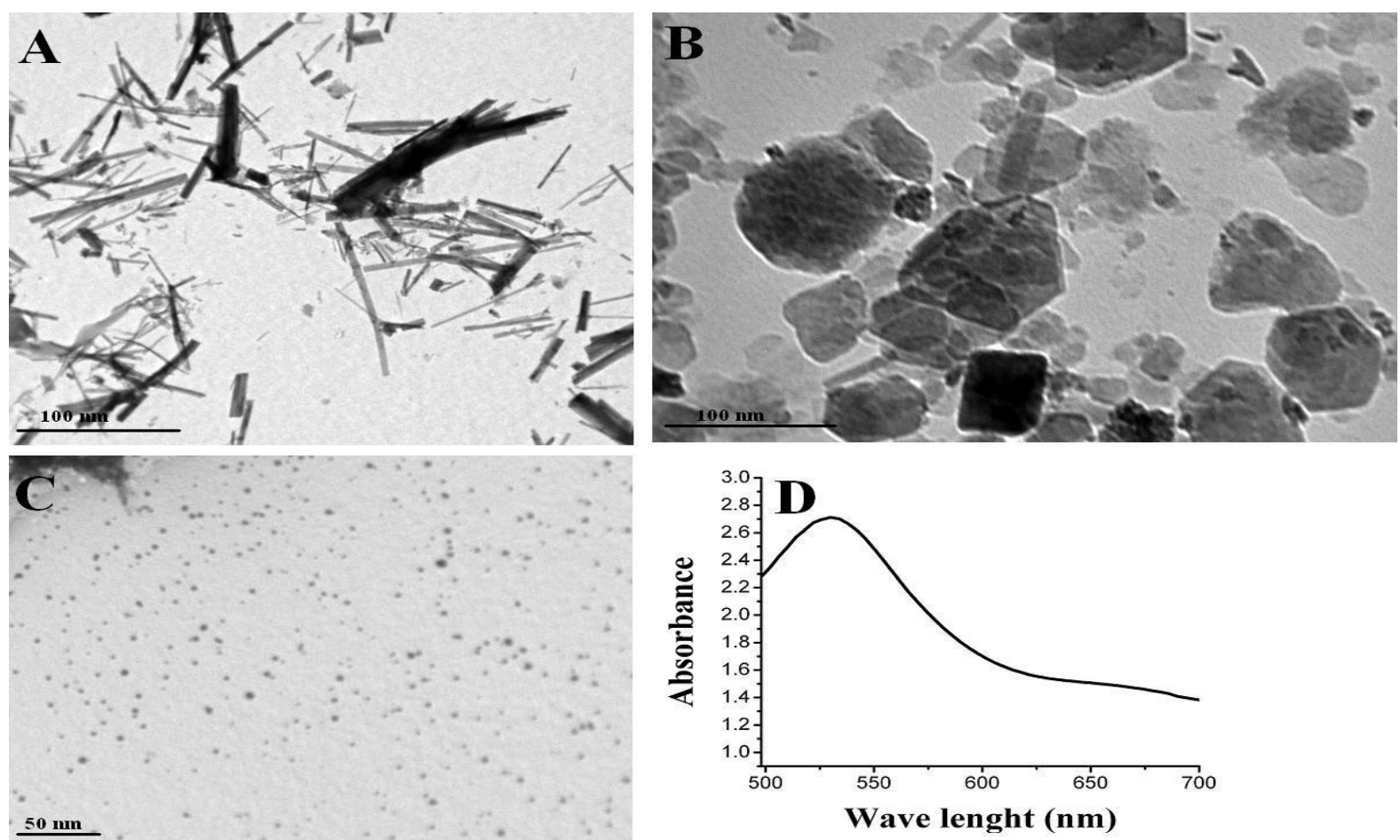

Figure 1. SEM morphologies of the haudroxyapatite nHA (A), tri-calcuim phosphate (B) and gold nanoparticles (Au NPs) (C) and UV absorbance for the Au NPs (D
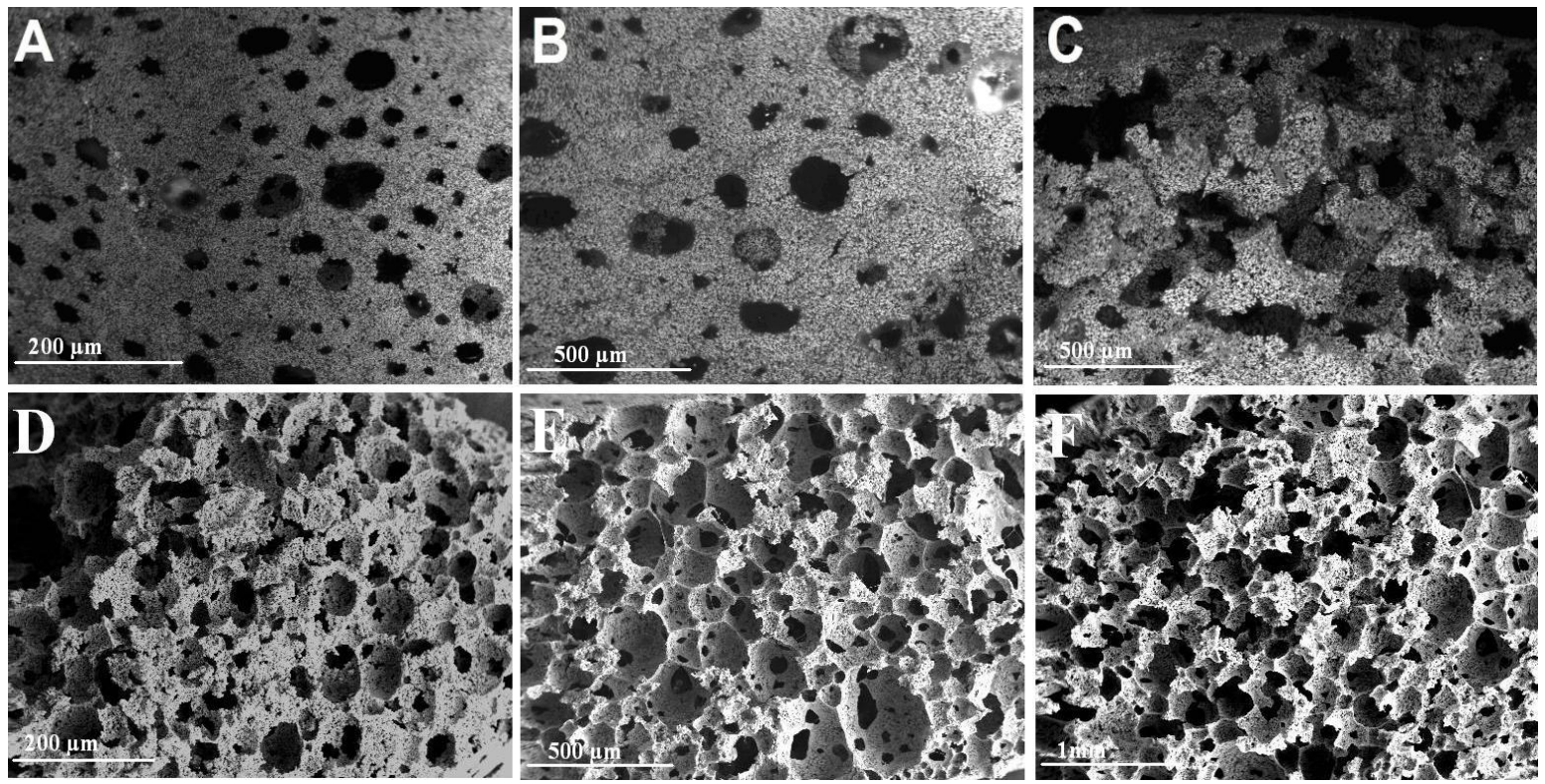

Figure 2. SEM morphologies of the ceramic scaffolds: (A) HA-TCP (60\%), (B) HA-TCP (50\%), and (C) HA-TCP (20\%); SEM of Au loaded ceramic scaffold: (E) HA-TCP-Au NPs (60\%), (F) HA-TCP-Au NPs (50\%), and (G) HATCP-Au NPs $(20 \%)$. 

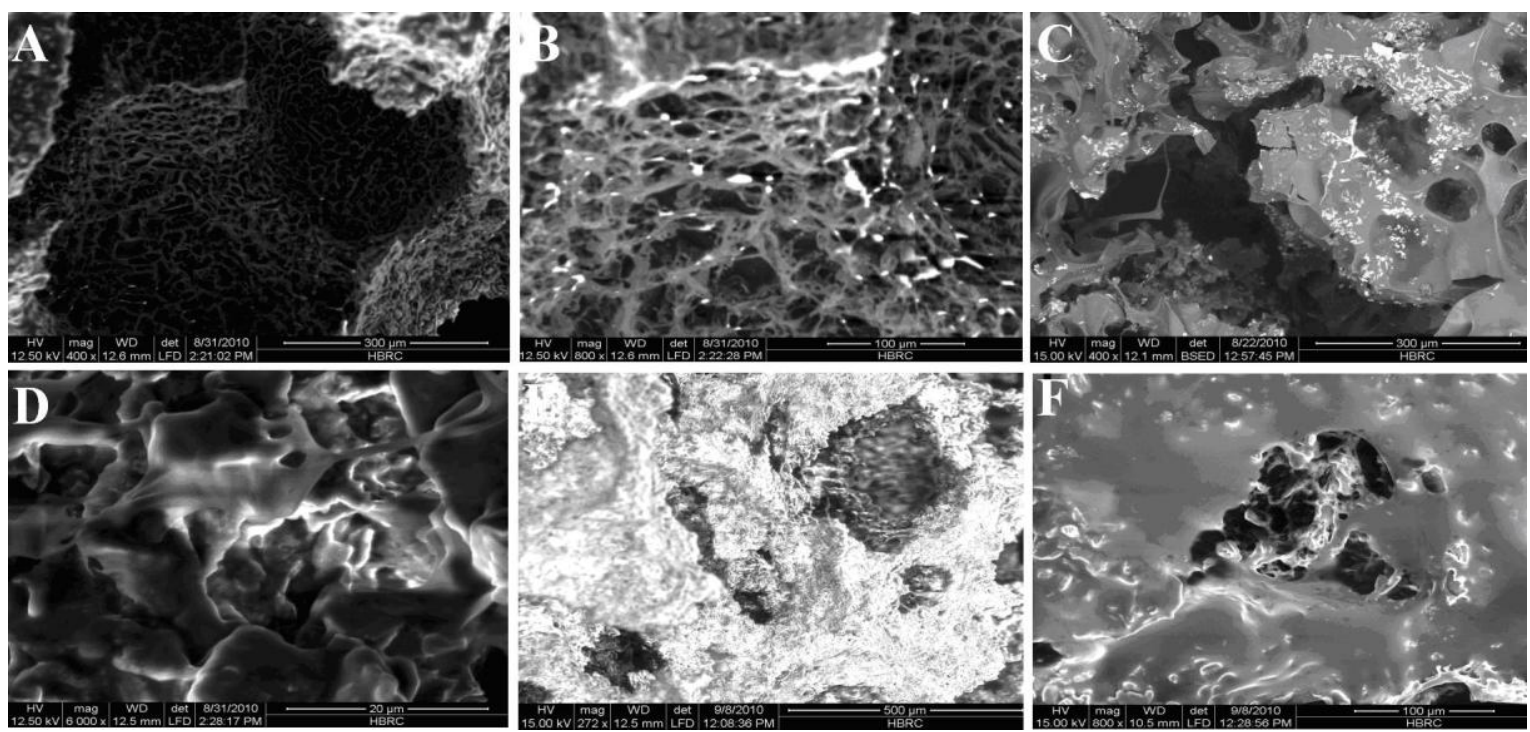

Figure 3. SEM of Au loaded scaffold with osteoblast cells on day 14 (A, B), day 21 (C,D) and day 26 (E,F).

The Au NPs have no toxic effects on the cells viability this may be due to the enhanced interaction between Au NPs and growing cells on the biopolymer matrix. Au NPs may develop London - Van Der-Waals forces with cells. Additionally, the Au NPs can act as adhesive between biopolymer and cells.

\subsection{In vitro Degradation Test}

To support the differentiation and proliferation of cells, a scaffold should be biodegradable and bioactive. The degradability of the ceramic and Au NPs loaded ceramic scaffold was monitored at 3, 5, 10, 15, 25 and 30 days in culture, as shown in Fig. 4-A, B.
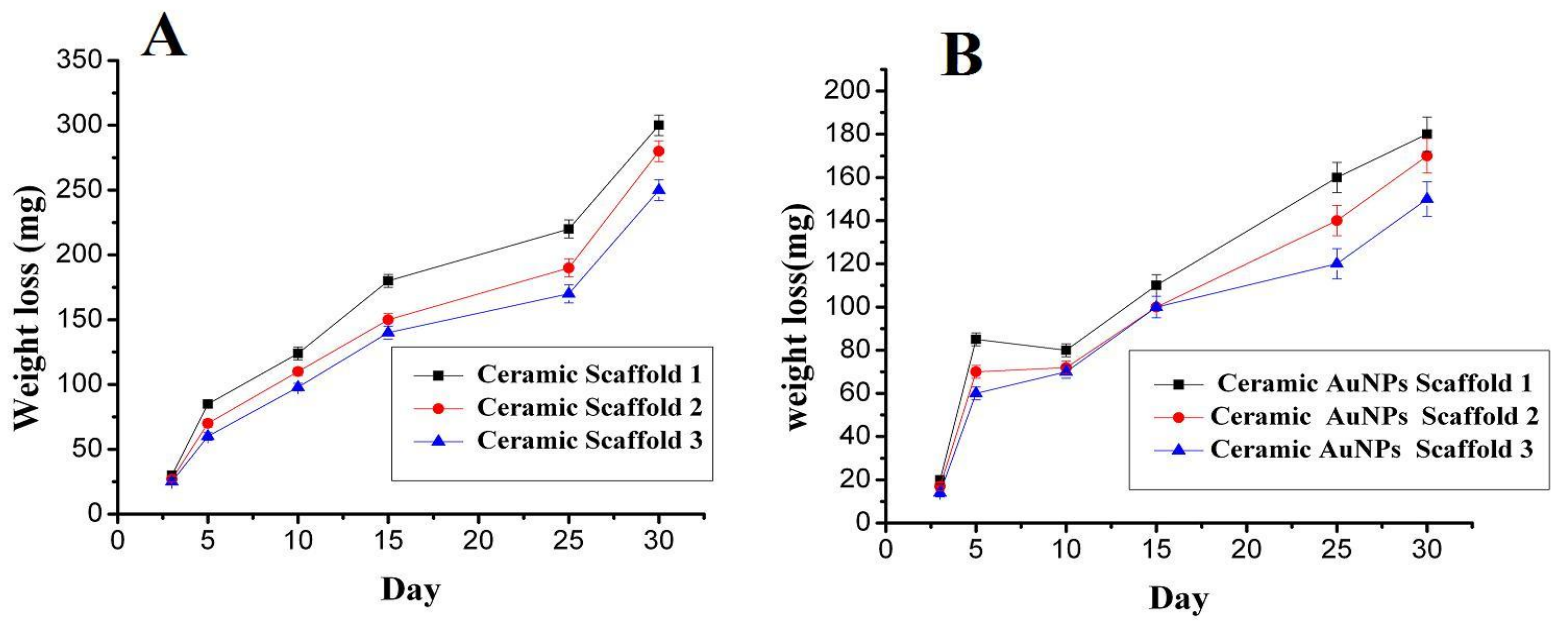

Figure 4. The rate of degradation of ceramic scaffold (A), with different concentrations of HA-TCP and ceramic scaffold loaded with Au NPs and (B), with varying concentrations of HA-TCP-Au NPs.

Apparently, the degradation rates of Au NPs loaded ceramic scaffold increased gradually and the weight loss ranged between $20-180 \mathrm{mg}$. Briefly, the weight loss ranged between $20-85 \mathrm{mg}$ at day 3,5 , whereas it decreased at day 15 ranging between $75-80 \mathrm{mg}$, followed by a steady increase in the rate degradation up till day 30, ranging between $25-300 \mathrm{mg}$. The decrease in degradation rate at day 15 can be explained by the gold ceramic powder depositing and partially saturating the SBF solution. Also, the increasing degradation rates after day 15 may be attributed to decreasing 
powder concentrations. These result indicate that degradation rates of ceramic is faster than that in $\mathrm{Au}$ NPs loaded ceramic scaffold, due to Au NPs delaying degradation via decreasing the solubility of the scaffold. Furthermore, the results clearly indicate that the Au NPs loaded ceramic scaffold can induce powder particles to deposit in SBF, and thus has a better bioactivity in vitro, compared to the ceramic scaffolds. As a result, it is expected that $\mathrm{Au}$ loaded scaffold will have the ability to make a direct bond to living bone when implanted in the body, because of the resemblance of $\mathrm{Au}$ NPs ceramic ion concentrations of SBF solution with that of blood plasma [32-33].

\subsection{Scaffold Porosity}

The porosity percentage of the ceramic and ceramic scaffold loaded with Au NPs are shown in Fig. 5, which show the ceramic scaffolds porosity ranging between $30-55 \%$ and its porosity decreasing with increasing ceramic powder concentration. On the other hand, Au NPs loaded ceramic scaffold displayed porosity ranging between $50-80 \%$, which is significantly larger than that of the ceramic scaffold for the same powder concentration and was also, evident in the SEM results. These results clearly indicate that the porosity of ceramic and Au NPs loaded ceramic scaffold is inversely proportional with powder concentration. The increased porosity of $\mathrm{Au}$ loaded scaffold compared to ceramic scaffold may be attributed to the large surface area of the $\mathrm{Au}$ NPs, which increased particles interaction and enhanced thermal sintering via exothermic heat evolved from $\mathrm{Au} \mathrm{NPs}$ at $280^{\circ} \mathrm{C}$. The structural advantages of ceramic scaffolds loaded with $\mathrm{Au}$ NPS include high porosity, high volume-to-area ratios, high surface area, and high structural stability due to unique properties of $\mathrm{Au}$ NPs $<10 \mathrm{~nm}$. Thereby, enhancement of porosity upon addition of ceramic scaffold loaded with Au NPs may $\mathrm{Au}$ NPs could act as non solvent.Therefore, $\mathrm{Au}$ NPs loaded ceramic scaffold is more effective than ceramic scaffold for tissue engineering applications because of its higher porosity, which enhances cell growth and differentiations [32].

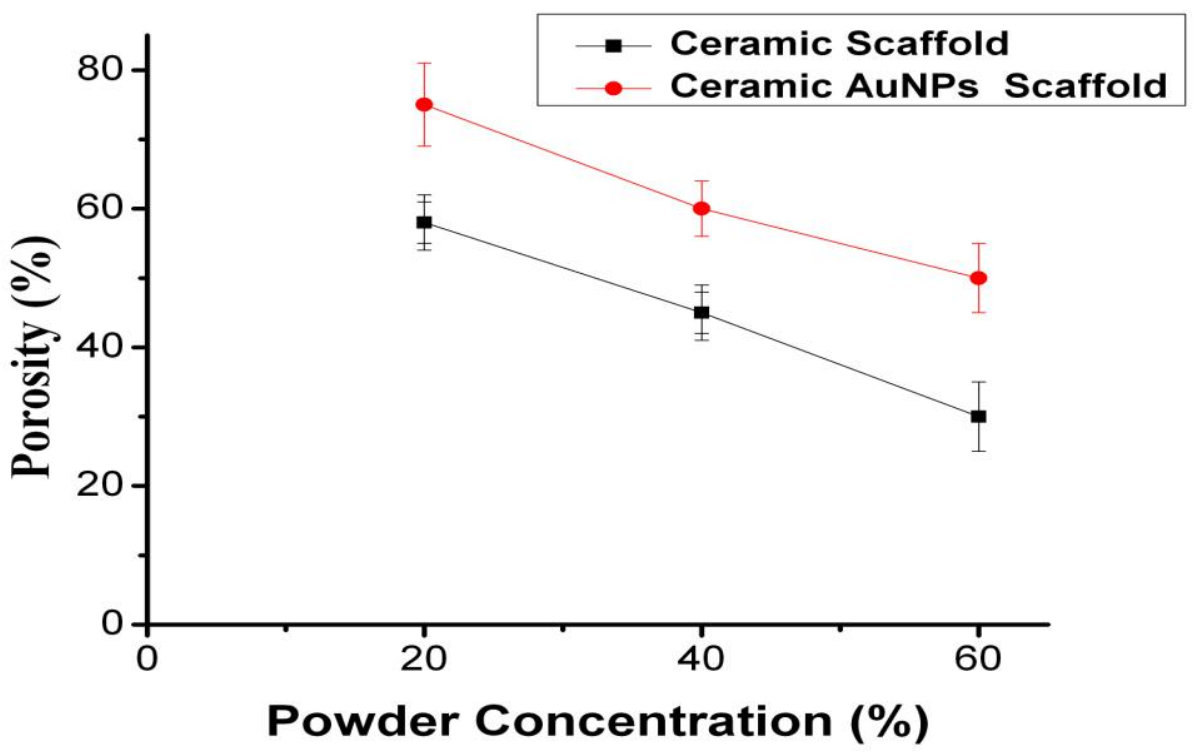

Figure 5. The porosity percentage for Ceramic scaffold and ceramic scaffold loaded with Au NPs.

\subsection{Mechanical property analysis}

Fig. 6 shows the relation between the ceramic powder concentration and compressive strength of ceramic and $\mathrm{Au}$ NPs loaded ceramic scaffold, which demonstrates that the scaffolds compressive strength varied proportionally with changing of the powder concentrations. Briefly, the scaffolds compressive strength was increased with increasing both of the ceramic and ceramic loaded with $\mathrm{Au}$ NPs concentration. The compressive 
strength ranged between $12-30 \mathrm{MPa}$ for ceramic scaffold and ranged between $25-45 \mathrm{MPa}$ for $\mathrm{Au}$ NPs loaded ceramic scaffold. The results clearly demonstrate that the scaffold compressive strength is enhanced through addition of $\mathrm{Au}$ NPs to ceramic scaffold. This may be explained by the higher surface area of Au NPs, which could have enhanced the scaffold ability to self reinforce during setting in humid condition rather than ceramic scaffold. The higher compressive strength of $\mathrm{Au}$ NPs loaded ceramic scaffold underscore their superiority, compared to ceramic scaffold, for implantation in organs that require high load bearing, such as femurs, tibia and foot [32].

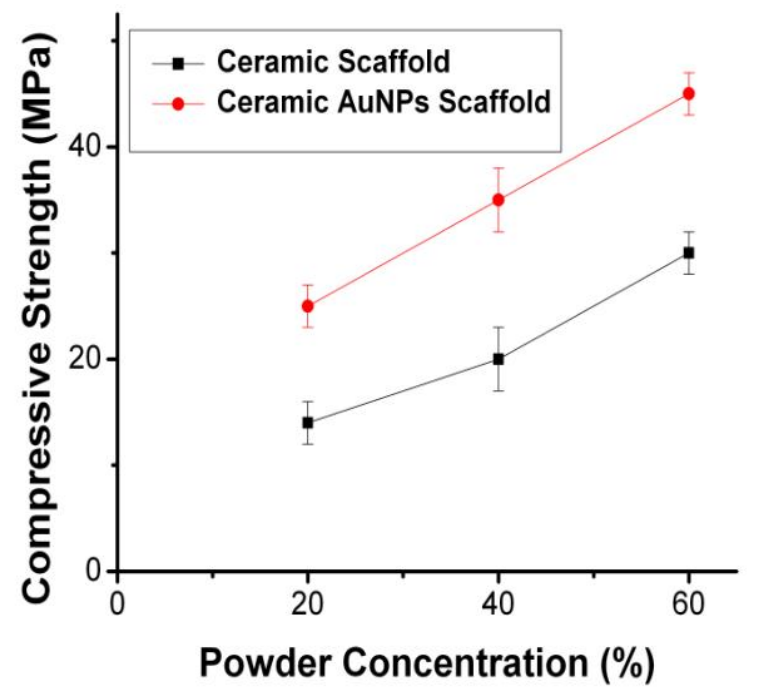

Figure 6. Compressive strength of ceramic and $\mathrm{Au}$ loaded ceramic scaffolds with various powder concentrations.

\section{Conclusions}

Currently, there are number of strategies for developing bone tissue engineering. One of the most unique of these strategies is ceramic scaffold nanocomposites, which have successfully achieved bioactivity, degradability, and suitable mechanical properties compared to conventional polymeric scaffolds. Herein we fabricated ceramic scaffold and Au loaded ceramic scaffold with acceptable mechanical, degradability, and bioactivity properties. The effects of the varying ceramic powder concentration, ceramic Au NPs on the scaffold morphology, porosity, degradability, mechanical properties and cells viability has been studied. The ceramic scaffold's porosity were ranged between $30-58 \%$ and pore diameters were in the range of of $50-400 \mu \mathrm{m}$, while compressive strength ranged between $12-30 \mathrm{MPa}$. Conversely, porosity of $\mathrm{Au}$ NPs loaded ceramic scaffold ranged between $50-80 \%$ with pore diameter ranging between $100-600 \mu \mathrm{m}$ and compressive strength in range of $25-45 \mathrm{MPa}$. Porosity and mechanical strength of $\mathrm{Au}$ loaded ceramic scaffolds was shown to be significantly higher than that of the ceramic scaffold for the same powder concentration. Additionally, porosity decreased as ceramic powder concentrations increased, whereas scaffold compressive strength was enhanced by increasing ceramic concentration. Degradation rates of ceramic scaffold ranged between $25-300 \mathrm{mg}$ during an experiment duration of 3-30 days, while degradation rates for the Au NPs loaded ceramic scaffold ranged between 20-180mg. Most interestingly, osteoblast cells grew, differentiated and formed sheet network after 14 day of seeding in the $\mathrm{Au}$ NPs loaded ceramic scaffold, and all pores were completely covered with cells after 26 day of culture. These results clearly reveal that $\mathrm{Au}$ NPs have noted effects on the scaffold porosity, degradation rates, compressive strength and posses suitable support for the cells differentiation and have compressive strength similar to that of calceneous bone. To our knowledge, metallic $\mathrm{Au}$ NPs have not been incorporate previously with calcium phosphate for fabrication scaffold for autologus bone grafting. Also we are the first group studied the effects of $\mathrm{Au}$ NPS on the mechanical properties, porosity and degradation rates for scaffolds.

In conclusion, the current study clearly demonstrates that the incorporation of $\mathrm{Au}$ NPs with ceramic powder for scaffold fabrication could be an active, safe and inexpensive tool for implantations in organs that need high bearing load such as femurs, tibia and foot. This may eliminate the need for metal implantation, which is always associated with infection and the suffering of repeat surgeries. 


\section{References}

1. Ballo, A., et al., Bone tissue responses to glass fiber - reinforced composite implants-a histomorphometric study. Clinical oral implants research, 2009. 20(6): p. 608-615. doi: 10.1111/j.1600-0501.2008.01700.x

2. Laurencin, C., Y. Khan, and S.F. El Amin,Bone graft substitutes. Expert review of medical devices, 2006. 3(1): p. 49-57. doi: 10.1586/ 17434440.3.1.49

3. Wang, X., et al., Fabrication and characterization of porous hydroxyapatite/ $\beta$ tricalcium phosphate ceramics by microwave sintering. Materials Letters, 2006. 60(4): p. 455-458. doi:10.1016/j.matlet.2005.09.010

4. Roy, M., et al., Laser processing of bioactive tricalcium phosphate coating on titanium for load-bearing implants. Acta Biomaterialia, 2008. 4(2): $\quad$ p. 324-333. doi:10.1016/j.actbio.2007.09.008

5. Jensen, S.S., et al., Bone healing and graft resorption of autograft, anorganic bovine bone and $\beta$-tricalcium phosphate. A histologic and histomorphometric study in the mandibles of minipigs. Clinical oral implants research, 2006. 17(3): p. 237-243. DOI: 10.1111/j.16000501.2005.01257.x

6. Miao, X., et al., Mechanical and biological properties of hydroxyapatite/tricalcium phosphate scaffolds coated with poly (lacticco-glycolic acid). Acta Biomaterialia, 2008. 4(3): p. 638-645. doi: 10.1016/j.actbio. 2007.10.006

7. Woodard, J.R., et al., The mechanical properties and osteoconductivity of hydroxyapatite bone scaffolds with multi-scale porosity. Biomaterials, 2007. 28(1): p. 45-54. doi: 10.1016/j.biomaterials.2006.08.021

8. Lee, H., et al., Designed hybrid scaffolds consisting of polycaprolactone microstrands and electrospun collagen - nanofibers for bone tissue regeneration. Journal of Biomedical Materials Research Part B: Applied Biomaterials, 2011. 97(2): p. 263-270. DOI: 10.1002/jbm.b.31809

9. Mathieu, L.M., et al., Architecture and properties of anisotropic polymer composite scaffolds for bone tissue engineering.
Biomaterials, 2006. 27(6): p. 905-916. doi: 10.1016/j.biomaterials.2005.07.015

10. Wang, H., et al., Biocompatibility and osteogenesis of biomimetic nanohydroxyapatite/polyamide composite scaffolds for bone tissue engineering. Biomaterials, 2007. 28(22): p. 3338-3348. doi. 10.1016/ j.biomaterials.2007.04.014

11. Shor, L., et al., Fabrication of threedimensional polycaprolactone/hydroxyapatite tissue scaffolds and osteoblast-scaffold interactions in vitro. Biomaterials, 2007. 28(35): p. 5291-5297. doi. 10.1016/ j.biomaterials.2007.08.018

12. Hollister, S., R. Maddox, and J. Taboas, Optimal design and fabrication of scaffolds to mimic tissue properties and satisfy biological constraints. Biomaterials, 2002. 23(20): p. 4095-4103. doi: 10.1016/S0142-9612(02) 00148-5

13. Eid, K.A.M. and H.M.E. Azzazy, Controlled synthesis and characterization of hollow flower-like silver nanostructures. International Journal of Nanomedicine, 2012. 7: p. 15431550. doi: 10.2147/IJN.S26524

14. HF, S., E. KAM, and S. MA, Formulation and evaluation of silver nanoparticles as antibacterial and antifungal agents with a minimal cytotoxic effect. International Journal of Drug Delivery, 2011. 3(2): p. 293-304. doi: $10.5138 /$ ijdd.v3i2.224

15. Ou, J., et al., Uniform polystyrene microspheres decorated with noble metal nanoparticles formed without using extra reducing agent. Colloids and Surfaces A: Physicochemical and Engineering Aspects, 2007. 305(1-3): p. 36-41. doi: 10.1016/ j.colsurfa.2007.04.038

16. Storhoff, J.J., et al., Gold nanoparticle-based detection of genomic DNA targets on microarrays using a novel optical detection system. Biosensors and Bioelectronics, 2004. 19(8): p. 875-883.

17. Jain, P.K., et al., Noble metals on the nanoscale: optical and photothermal properties and some applications in imaging, sensing, biology, and medicine. Accounts of Chemical Research, 2008. 41(12): p. 15781586. DOI: $10.1021 / \operatorname{ar} 7002804$ 
18. Eid, K.A.M., et al., Antifungal Effects of Colloidally Stabilized Gold Nanoparticles: Screening by Microplate Assay. nature, 2011. 2: p. 9. doi: 1545-0740

19. Arumugam, S.K., et al., Formation of gold nanoparticles on hydroxyapatite surface for enhancement of blood compatibility via a negative cilia concept. Journal of Biomedical Nanotechnology, 2006. 2(1): p. 46-52. doi: 10.1166/jbn.2006.009

20. Thevenot, P., W. Hu, and L. Tang, SURFACE CHEMISTRY INFLUENCE IMPLANT BIOCOMPATIBILITY. Current topics in medicinal chemistry, 2008. 8(4): p. 270-280. doi: $10.2174 / 156802608783790901$

21. Horii, A., et al., Biological designer selfassembling peptide nanofiber scaffolds significantly enhance osteoblast proliferation, differentiation and 3-D migration. PLoS One, $2007 . \quad 2(2): \quad$ p. doi:10.1371/journal.pone.0000190

22. Kimling, J., et al., Turkevich method for gold nanoparticle synthesis revisited. The Journal of Physical Chemistry B, 2006. 110(32): p. 15700-15707. DOI: 10.1021/jp061667w

23. Kithva, P.H., et al., An organic matrixmediated processing methodology to fabricate hydroxyapatite based nanostructured biocomposites. Nanoscale, 2009. 1(2): p. 229232. DOI: $10.1039 / B 9 N R 00062 C$

24. Kong, L., J. Ma, and F. Boey, Nanosized hydroxyapatite powders derived from coprecipitation process. Journal of materials science, 2002. 37(6): p. 1131-1134. DOI: $10.1023 / \mathrm{A}: 1014355103125$

25. Cuneyt Tas, A., et al., An investigation of the chemical synthesis and high-temperature sintering behaviour of calcium hydroxyapatite (HA) and tricalcium phosphate (TCP) bioceramics. Journal of Materials Science:
Materials in Medicine, 1997. 8(2): p. 91-96. DOI: $10.1023 / \mathrm{A}: 1018506800033$

26. Zhang, Y. and M. Zhang, Synthesis and characterization of macroporous chitosan/calcium phosphate composite scaffolds for tissue engineering. Journal of biomedical materials research, 2001. 55(3): p. 304-312. DOI: 10.1002/1097-4636

27. So, K., et al., Accelerated degradation and improved bone-bonding ability of hydroxyapatite ceramics by the addition of glass. Biomaterials, 2006. 27(27): p. 47384744. doi: 10.1016/j.biomaterials.2006.05.020

28. Sobhana, S.S.L., et al., Gelatin-Chitosan composite capped gold nanoparticles: a matrix for the growth of hydroxyapatite. Journal of Nanoparticle Research, 2009. 11(2): p. 333-340. DOI: 10.1007/s11051-008-9385-0

29. Podsiadlo, P., et al., Gold nanoparticles enhance the anti-leukemia action of a 6mercaptopurine chemotherapeutic agent. Langmuir, 2008. 24(2): p. 568-574. DOI: $10.1021 / 1 \mathrm{a} 702782 \mathrm{k}$

30. Kim, K., et al., Photoacoustic imaging of early inflammatory response using gold nanorods. Applied physics letters, 2007. 90: p. 223901. DOI: $10.1063 / 1.2743752$

31. Rautaray, D., S. Mandal, and M. Sastry, Synthesis of hydroxyapatite crystals using amino acid-capped gold nanoparticles as a scaffold. Langmuir, 2005. 21(11): p. 51855191. DOI: $10.1021 / \mathrm{la048541f}$

32. Huang, J., et al., In vitro mechanical and biological assessment of hydroxyapatitereinforced polyethylene composite. Journal of Materials Science: Materials in Medicine, 1997. 8(12): p. 775-779. doi: 10.1023/ $\underline{\text { A: } 1018516813604}$ 